\title{
Severe Phenotype of Phosphorylase Kinase-Deficient Liver Glycogenosis with Mutations in the PHKG2 Gene
}

\author{
BARBARA BURWINKEL, TERJE ROOTWELT, ELI ANNE KVITTINGEN, \\ PRANESH K. CHAKRABORTY, AND MANFRED W. KILIMANN \\ Institut für Physiologische Chemie, Medizinische Fakultät, Ruhr-Universität Bochum, D-44780 Bochum, \\ Germany [B.B., M.W.K.]; Departments of Pediatrics [T.R.] and Clinical Chemistry [E.A.K.], \\ Rikshospitalet, N-0027 Oslo, Norway; and Division of Clinical and Metabolic Genetics, The Hospital for \\ Sick Children, Toronto, Ontario M5G 1X8, Canada [P.K.C.]
}

\begin{abstract}
Phosphorylase kinase-deficient liver glycogenosis manifests in infancy with hepatomegaly, growth retardation, and elevated plasma aminotransferases and lipids. It can be caused by mutations in three different genes of phosphorylase kinase subunits: $P H K A 2, P H K B$, and $P H K G 2$. It is usually a benign condition, often with complete resolution of symptoms during puberty. A minority of patients displays a more severe phenotype with symptomatic fasting hypoglycemia and abnormal liver histology that may progress to cirrhosis. Three patients with liver cirrhosis in childhood analyzed previously all had $P H K G 2$ mutations. This suggested that this genotype may generally cause a more severe clinical manifestation, but to date $P H K G 2$ mutations have been identified in only seven patients. Here, we report mutation analysis in three new patients with liver phosphorylase kinase deficiency and recurrent hypoglycemia, liver fibrosis, and lack of glucagon response but no overt cirrhosis. In all three patients,
\end{abstract}

\section{ABSTRACT}

PHKG2 mutations were found (H89fs[insC], E157K, D215N, W300X). Three of these mutations are novel, bringing the total number of distinct human PHKG2 mutations to 11 , found in 10 patients. We conclude that liver phosphorylase kinase deficiency with a severe phenotype, with or without cirrhosis, is indeed often caused by $P H K G 2$ mutations. These patients require active measures to maintain normoglycemia (raw cornstarch, nocturnal tube feeding), which may also alleviate growth retardation and the development of abnormal liver histology. (Pediatr Res 54: 834-839, 2003)
ALT, alanine transaminase
GSD, glycogen storage disease
Phk, phosphorylase kinase (E.C. 2.7.1.38)
XLG, X-linked liver glycogenosis

GSDs (glycogenoses) are caused by genetic deficiencies of various enzymes or transporters involved in the metabolism of glycogen or glucose. Depending on the mutant protein and its tissue distribution, most glycogenoses manifest primarily in liver, in muscle, or in both. GSDs of the liver occur with a collective frequency of approximately 1 in 25,000 births in Europe (1).

Deficiency of Phk probably constitutes the largest subgroup of liver glycogenoses (frequency, approximately 1 in 100,000 births). Phk is a protein kinase that mediates the neural and

Received October 22, 2002; accepted May 7, 2003.

Correspondence: Manfred W. Kilimann, M.D., Institut für Physiologische Chemie, RuhrUniversität Bochum, D-44780 Bochum, Germany; e-mail: manfred.kilimann@ ruhr-uni-bochum.de

Present address (MWK): Department of Cell and Molecular Biology, Uppsala University, BMC Box 596, SE-75124 Uppsala, Sweden; e-mail: manfred.kilimann@icm.uu.se

Supported by the Deutsche Forschungsgemeinschaft and the Fonds der Chemischen Industrie.

DOI: 10.1203/01.PDR.0000088069.09275.10 hormonal regulation of glycogen breakdown by glycogen phosphorylase. Phk is a heterotetrameric protein [subunit structure, $\left.(\alpha \beta \gamma \delta)_{4}\right]$. Moreover, each subunit has tissue-specific isoforms or splice variants. Phk deficiency is therefore genetically and clinically heterogeneous, occurring in several variants that differ in tissue involvement and mode of inheritance [Table 1; reviewed by Kilimann (2)]. A glycogenosis of the liver can be caused by mutations in any one of three Phk genes. The most common are mutations of the $\alpha$ subunit liver isoform gene, PHKA2, causing an X-linked liver-specific variant (3-6). Mutations in the gene of the ubiquitously expressed $\beta$ subunit, $P H K B$, give rise to an autosomal-recessive glycogenosis of liver and muscle (7-9), whereas mutations in the gene of the testis/liver isoform of the $\gamma$ subunit, $P H K G 2$, are responsible for an autosomal-recessive glycogenosis of liver alone (1013). Phk deficiency in these disorders can also be detected in erythrocytes, leukocytes, and fibroblasts, except in patients with certain PHKA2 missense mutations (XLG subtype 2, 
Table 1. Subtypes of Phk-deficient GSDs

\begin{tabular}{cll}
\hline \multicolumn{1}{c}{ Inheritance } & \multicolumn{1}{c}{ Tissues affected } & \multicolumn{1}{c}{$\begin{array}{c}\text { Mutant } \\
\text { gene }\end{array}$} \\
\hline X-linked recessive & muscle & PHKA1 \\
& liver* $(X L G 1)$ & $P H K A 2$ \\
& liver (blood cells normal; $X L G 2)$ & $P H K A 2$ \\
Autosomal recessive & liver* and muscle & PHKB \\
& liver* & PHKG2 \\
& muscle & unidentified \\
& heart & unidentified \\
\hline
\end{tabular}

* Phk deficiencies affecting the liver are also detectable in blood cells, with the exception of the $X L G 2$ group.

XLG2) $(3,5)$. In addition, muscle-specific and heart-specific forms of Phk deficiency are known (Table 1) $(1,2)$.

Compared with other types of liver glycogenosis, Phk deficiency is usually a mild condition. Most patients present as infants with hepatomegaly, growth retardation, and moderately raised plasma lipids and aminotransferases. In contrast to GSD type I, lactic acidosis and clinically apparent hypoglycemia are observed only in a minority. Hepatic architecture typically remains normal, unlike GSD type III, in which fibrosis is common, and unlike GSD type IV, which usually progresses to cirrhosis in childhood. The course of Phk deficiency is also usually benign, such that hepatomegaly often recedes and patients catch up to normal height after puberty $(1,14)$. Muscle weakness may or may not be observed with any genotype. $P H K B$-mutant patients can be without muscle symptoms although Phk is deficient in this tissue, whereas PHKA2- or $P H K G 2$-mutant patients may display muscle weakness in spite of normal muscle Phk activity. Growth retardation and weakness could be the result of chronically low systemic glucose and consequent hypoinsulinism, as in GSD I $(1,15,16)$.

However, some patients with Phk deficiency have more severe symptoms, and emerging data suggest a genotypephenotype correlation. The functional impact of $P H K B$ mutations, both on residual enzyme activity and clinical condition, appears to be the mildest (7-9). PHKA2 mutations, which are most common, are usually associated with a benign disease although more severe manifestations are also observed (3-6, 14). PHKG2 mutations have so far been identified in only seven patients. These cases seem to concentrate at the other end of the spectrum of severity, with very low residual Phk activities, particularly high plasma lipids and aminotransferases, and other abnormalities that are uncommon in Phk deficiency such as frequent fasting hypoglycemia, an impaired glucagon response, hepatocellular adenoma, or fibrosis. Most strikingly, the only three published cases of patients with Phk deficiency who developed liver cirrhosis in childhood were all found to carry PHKG2 mutations $(10-13,17)$.

To test the apparent correlation between PHKG2 mutations and a severe phenotype, also in cases without overt cirrhosis, we analyzed three additional liver Phk-deficiency patients with unusually severe symptoms (frequent episodes of hypoglycemia, absent glucagon response, and abnormal liver histology). PHKG2 mutations were found in all three patients.

\section{METHODS}

Case descriptions. Patient 1, female, is the first child of healthy, distantly related Norwegian parents. A large, firm abdomen was noted since birth, and hepatomegaly was observed at 9 mo. Signs of hypoglycemia (somnolence, eye deviation, and lack of contact) were noted repeatedly, and plasma glucose values of $1.6-2.3 \mathrm{mM}$ and ketosis were determined on fasting. Plasma triglycerides and cholesterol were slightly increased (1.3-2.2 and 3.5-4.0 mM, respectively), whereas liver enzymes were markedly elevated (aspartate transaminase and ALT, 250-2000 U/L). Uric acid was repeatedly normal. Resting lactate was normal $(0.7-1.7 \mathrm{mM})$ but rose to $3.2 \mathrm{mM}$ after glucose load and to $7.7 \mathrm{mM}$ after galactose [glucose and galactose given orally at $2 \mathrm{~g} / \mathrm{kg}$ (18)]. Liver histology at 10 mo of age showed massive cytoplasmic glycogen storage and moderate fibrosis. In erythrocytes, Phk activity was low (4.8 $\mu \mathrm{mol} \mathrm{P}_{\mathrm{i}} \cdot \mathrm{g} \mathrm{Hb} \mathrm{Hb}^{-1} \cdot \mathrm{min}^{-1}$; normal, 10-90) and glycogen very high (449 $\mu \mathrm{g} / \mathrm{g} \mathrm{Hb}$; normal, 10-120). Total phosphorylase, $\alpha$-glucosidase, and debranching enzyme in leukocytes were normal, whereas the phosphorylase \pm AMP activity ratio was decreased (0.15; normal ratio, $0.42-0.78)$. Two glucagon tests $(0.03 \mathrm{mg} / \mathrm{kg}$ i.v. $)$ produced no rise in glucose. Muscle histology, glycogen content, and Phk activity were normal. Growth has been slow, with length $3 \mathrm{~cm}$ below the 2.5 th percentile until $4 \mathrm{y}$, and length at the 10th percentile and weight at the 50th percentile at $8 \mathrm{y}$ of age. She is an active girl with no signs of muscle weakness. Nocturnal gastric tube feeding (2-3 $\mathrm{mg} \cdot \mathrm{kg}^{-1} \cdot \mathrm{min}^{-1}$ of glucose polymer) was started at 1 y $3 \mathrm{mo}$ and is continued to date ( $8 \mathrm{y})$ in combination with daytime feeding of uncooked cornstarch $(2 \mathrm{~g} / \mathrm{kg}$ per day $)$. The hypoglycemic tendency persists: at the age of 6 years a 4-h fast resulted in a plasma glucose of $2.3 \mathrm{mM}$. Aminotransferases continue to be markedly elevated whereas clotting and bilirubin are normal. The patient needs supplementation of fatsoluble vitamins; without this her vitamin D levels were repeatedly subnormal. Ultrasound at $8 \mathrm{y}$ of age showed marked hepatomegaly (10 $\mathrm{cm}$ below costal margin) with a coarse nodular echo and a slightly enlarged spleen. Both kidneys were of normal size $(8 \mathrm{~cm}$; normal range, $7-10 \mathrm{~cm})$. Doppler flows were normal and there were no other signs of portal hypertension.

Patient 2, female, is the second child of unrelated Norwegian parents. The parents and brother are unaffected. She came to our attention with repeated seizures from 4 mo of age. Marked hepatomegaly was noted, and hypoglycemia around $2 \mathrm{mM}$ was repeatedly determined. Plasma cholesterol and uric acid were normal, and aminotransferases were repeatedly at 300-500 $\mathrm{U} / \mathrm{L}$. Liver histology at 11 mo revealed glycogen deposition and mild fibrosis. Erythrocyte glycogen was $8500 \mu \mathrm{g} / \mathrm{g} \mathrm{Hb}$ (normal, 10-120), and erythrocyte Phk was $10 \mu \mathrm{mol} \mathrm{P}_{\mathrm{i}} \cdot \mathrm{g}$ $\mathrm{Hb}^{-1} \cdot \mathrm{min}^{-1}$ (normal, 10-90). Total phosphorylase and debranching enzyme activities in leukocytes were normal whereas the phosphorylase \pm AMP activity ratio was decreased $(0.21$; normal, $0.42-0.78)$. There was no response to glucagon $(0.03 \mathrm{mg} / \mathrm{kg}$ i.v.). Nighttime tube feeding was initiated at $11 \mathrm{mo}$ and continues to be necessary, as the patient suffered hypoglycemic seizures when the tube was discon- 
nected during the night at $6 \mathrm{y}$ of age. She is treated with cornstarch during the day $(2 \mathrm{~g} / \mathrm{kg}$ per day). Growth is markedly retarded, with her height consistently $4 \mathrm{~cm}$ below the 2.5 th percentile and her bone age 2 y delayed, but there are no signs of muscle weakness. At present (age, 7.5 years), aminotransferases continue to be elevated whereas clotting times and bilirubin are normal. A recent ultrasound showed marked hepatomegaly with diffusely increased echo, normal Doppler flows, normal kidney sizes $(8 \mathrm{~cm})$, and a slightly enlarged spleen.

Patient 3, female, is the third child of distantly related Chinese parents living in Trinidad. The parents, brother, and sister, and also the maternal grandfather and six maternal uncles, are unaffected, suggesting autosomal-recessive inheritance. Hepatomegaly was noted at 4 mo of age on routine physical examination. There was no history suggestive of hypoglycemia. Liver histology at 6 mo revealed marked glycogen accumulation plus portal and septal fibrosis. The glucose-6-phosphatase stain was normal. Plasma cholesterol, triglycerides, and uric acid were not elevated. Plasma ALT was elevated at $596 \mathrm{U} / \mathrm{L}$. A controlled fast at $14 \mathrm{mo}$ of age led to hypoglycemia $(1.9 \mathrm{mM})$ at $135 \mathrm{~min}$ of fasting with no lactic acidemia. There was no rise of glucose in response to glucagon administration $(0.025 \mathrm{mg} / \mathrm{kg}$ i.v. $)$. At $21 \mathrm{mo}$ of age she experienced episodes of nocturnal crying, sometimes associated with limb jerking, that responded to intake of juice or milk. Hypoglycemia was not documented during these episodes, but they resolved with supplemental uncooked cornstarch three times daily, which is continued to date $(24,24$, and $36 \mathrm{~g}$ at age, 7 y). Debranching enzyme activity in a second liver biopsy was normal; erythrocyte Phk activity was undetectable. At 4 y of age her spleen tip was first palpable. At 6 y of age, her spleen measured $6 \mathrm{~cm}$ below the costal margin. An abdominal ultrasound confirmed the hepatosplenomegaly, and Doppler studies showed normal unidirectional portal and hepatic venous blood flow. Mild thrombocytopenia was noted $\left(<100 \times 10^{9} / \mathrm{L}\right)$. Upper gastrointestinal endoscopy revealed one small esophageal varix. At $7 \mathrm{y}$ of age her splenomegaly persists but is less prominent. Doppler ultrasound continues to show unidirectional portal venous flow. Upper gastrointestinal endoscopy revealed an additional small esophageal varix. She has not experienced gastrointestinal bleeding or recent episodes of hypoglycemia. She is growing along the 50th centile for height, weight, and head circumference. She is developmentally appropriate, and has never had symptoms suggestive of muscle disease. Her mild thrombocytopenia has persisted. Her plasma aminotransferase activities have slowly decreased with time. Most recently, the aspartate transaminase was $364 \mathrm{U} / \mathrm{L}$ and the ALT was $189 \mathrm{U} / \mathrm{L}$. She has not been jaundiced. Her most recent conjugated bilirubin was elevated, however, at $17 \mu \mathrm{M}$.

Molecular analysis. Phk activity determinations for patients 1 and 2 were carried out according to Krebs (19) at the Department of Chemical Pathology, Great Ormond Street Hospital, London, U.K. Phk activity of patient 3 was measured according to Tuchman et al. (20) at the GSD Laboratory, Duke University Medical Center, Durham, NC, U.S.A.

Mutation analysis was performed under approval from the Review Board of the University of Bochum Medical School, and with informed consent of the subjects' parents. DNA was purified from patient blood, and all PHKG2 coding exons and flanking intron sequences were amplified by PCR and analyzed by direct sequencing as previously described (12).

Additional DNA samples were analyzed for the identified mutations by the technique of amplified created restriction sites, which introduces allele-specific restriction sites into PCR products by the use of primers that have one or two mismatches near the mutation. To detect mutation D215N, a sequence of 168 nucleotides was amplified with primers 5'-AAACCCACCCAGGCTATGGCAAGGTGATC (sense) and 5'-GCCAGCCAGGAGTGTGAAC (antisense). Two mismatches (in italics) in the sense primer together with the $\mathrm{D} 215 \mathrm{~N}$ mutation create a restriction site for $B c l \mathrm{I}$. The time protocol was $95^{\circ} \mathrm{C}$ for $5 \mathrm{~min}$ followed by 40 cycles of $95^{\circ} \mathrm{C} 10 \mathrm{~s}, 63^{\circ} \mathrm{C} 20 \mathrm{~s}$, and $72^{\circ} \mathrm{C} 20 \mathrm{~s}$, and finally $72^{\circ} \mathrm{C}$ for $5 \mathrm{~min}$, and the PCR products were digested with $B c l$ l. For analysis of mutation H89fs(insC), a sequence of 150 or 151 nucleotides was amplified with primers 5'-GCCACGAGTTTGCGGTGAAGA (sense) and 5'-CAAGGAAGACAGCCTCACCAATGTG (antisense). Two mismatches (in italics) in the antisense primer create a restriction site for $\mathrm{Xcm} \mathrm{I}$ in the normal sequence. The time protocol was $95^{\circ} \mathrm{C}$ for $7 \mathrm{~min}$ followed by 38 cycles of $94^{\circ} \mathrm{C} 30 \mathrm{~s}, 60^{\circ} \mathrm{C} 1$ min, and $72^{\circ} \mathrm{C} 20 \mathrm{~s}$, and finally $72^{\circ} \mathrm{C}$ for $15 \mathrm{~min}$, and the PCR products were digested with $X \mathrm{~cm}$ I. To detect mutation W300X, a sequence of 132 nucleotides across the mutation was amplified with primers 5'-TTTGAGCGTTGTGAAGGCAGCCAATCATG (sense) and 5'CAGGGTGAGCTCTAGTGCAG (antisense). Two mismatches (in italics) in the sense primer together with the W300X mutation create a restriction site for $B s p H$ I. The time protocol was $95^{\circ} \mathrm{C}$ for 5 min followed by 40 cycles of $95^{\circ} \mathrm{C} 10 \mathrm{~s}, 63^{\circ} \mathrm{C} 20 \mathrm{~s}$, and $72^{\circ} \mathrm{C} 20 \mathrm{~s}$, and finally $72^{\circ} \mathrm{C}$ for $5 \mathrm{~min}$, and the PCR products were digested with BspHI. All PCR reactions contained, in a total volume of $50 \mu \mathrm{L}, 4 \mu \mathrm{L}$ of DNA, $5 \mu \mathrm{L}$ of PCR buffer, $0.2 \mathrm{mM}$ of each dNTP, 20 pmol of each primer, 4, 3, or $6 \mu \mathrm{mol}$ of $\mathrm{MgCl}_{2}$, respectively, and 0.25 $\mu \mathrm{L}(5 \mathrm{U} / \mu \mathrm{L})$ AmpliTaq Gold polymerase (Applied Biosystems, Foster City, CA, U.S.A.). Products were electrophoresed on $3.5 \%$ NuSieve (Cambrex, East Rutherford, NJ, U.S.A.) agarose minigels and stained with ethidium bromide.

\section{RESULTS}

Patient 1 was found to be homozygous for a $G$ to $A$ transition converting aspartic acid 215 to asparagine (Fig. 1). D215 and its counterparts in other protein kinases, located in subdomain IX of the catalytic domain, is one of only 12 amino acid residues that are absolutely conserved in all protein kinases. It is apparently essential for the stabilization of the catalytic loop. This mutation has not been detected in humans before, but, remarkably, exactly the same mutation is responsible for the animal model of PHKG2-mutant Phk deficiency, the $g_{s} d$ rat (10).

Patient 2 was found to be compound heterozygous for two protein-truncating mutations (Fig. 1). The first is a novel, translation-terminating W300X nonsense mutation. Her second mutation is the insertion of an additional $\mathrm{C}$ residue into a tract of five cytosines, causing a frameshift beginning with histidine 
C A A GGAGGT CA A C C T GT GAGT

130

140

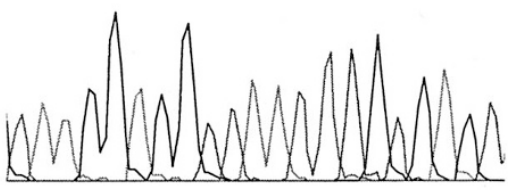

$\begin{array}{cccccc}\text { patient } 1 & \mathrm{~K} & \mathrm{E} & \mathrm{V} & \mathrm{D} & \mathrm{L} \\ \mathrm{AAG} & \mathrm{GAG} & \mathrm{GTC} & \mathrm{GAC} & \mathrm{CT} & \text { gtgagtt }\end{array}$

AAC

$\mathrm{N}$

C CAACC C T G N A ACCTCACCC C
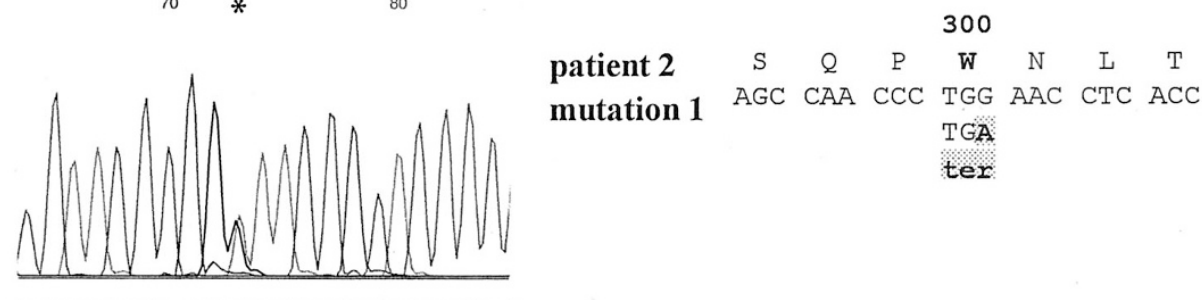

G G C C A C C C C C N N N N T A A N G N N

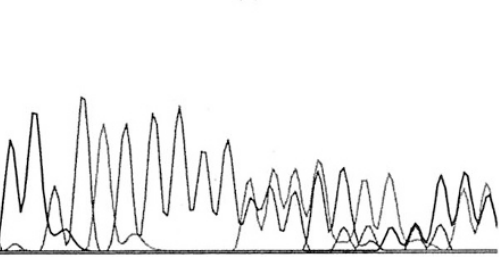

$\begin{array}{llllll}\text { patient } 2 & \text { G } & \text { H } & \text { P } & \text { H } & \text { I }\end{array}$

mutation 2 GGC CAC CCC CAC ATC A gtgagg

CCA CAT CA gegag

frameshift
Hum PHKG2

Rat PHKG2

Hum PHKG1

Rat PHKG1

Rbt PHKGI

Bov $\mathrm{cAPK} \alpha$

Bov $\mathrm{PKC} \alpha$

Yea CDC28
G $K E$ E D L W A C

$G K E V D L W A C$

$G K E V D M W$

$G K E V D M W S T$

G $K$ E V D M W $S$ T

$N$ K A V D W W A L

$G K S \quad V \quad D \quad W \quad W \quad A$

$S$ I $G$ D I W S I

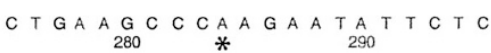
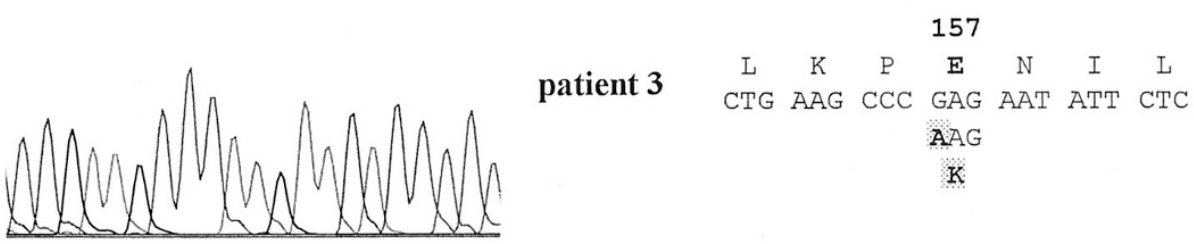

$D$ L $K$ P E N I L L D I $K$ K E N I L L $D$ L $K$ P E N I L L D L $\quad K \quad P$ E N I L L D L K P E N I L L $D$ L $K$ P E N L I I D L K L D N V M L D L K P \& N L L I

Figure 1. PHKG2 mutations in three patients with liver Phk deficiency associated with fasting hypoglycemia, no glucagon response, and liver fibrosis. Sequencer tracings are shown on the left, with mutant sequence positions indicated by asterisks (replacements) or an arrowhead (insertion). On the right, sequence alignments illustrate the conservation of missense-mutated amino acids in PHKG2 and PHKG1 from several species and in other protein kinases (bovine protein kinase A [ $\alpha$ isoform], bovine protein kinase C [ $\alpha$ isoform], and yeast CDC28 kinase). See www.sdsc.edu/kinases/pkr or Hanks and Quinn (24) for alignments of more protein kinase sequences.

89. This mutation was previously detected in another Norwegian patient (10).

Patient 3 was found to be homozygous for a novel $G$ to $A$ transition replacing glutamic acid 157 by a lysine residue (Fig. 1). E157 lies in subdomain VIb, implicated in ATP binding, of the catalytic domain. In an alignment of the catalytic domain sequences of 390 protein kinases (The Protein Kinase Resource, www.sdsc.edu/kinases/pkr), the corresponding position is occupied by acidic or polar neutral residues (mostly E, D, N, or Q) in other serine/threonine kinases, and either by alanine or arginine in tyrosine kinases. Lysine residues in this position are found exclusively in the activin-transforming growth factor $\beta$ receptor family, suggesting that this sequence feature is associated with a functional property highly specific for this kinase subfamily and inappropriate for Phk and all other protein kinases. The normal guanine nucleotide in this position is present in all other human PHKG2 sequences determined by us from 43 individuals, representing 80 independent chromosomes, and in all 60 expressed sequence tag and other cDNA sequences in the current sequence database. This indicates that $\mathrm{E} 157 \mathrm{~K}$ is not a common polymorphism, while occurring on both alleles of this patient who has consanguineous parents.

The mutations of patients 1 and 2 were confirmed, and additional family members analyzed for their presence, using the technique of amplified created restriction sites. This technique introduces allele-specific restriction sites into PCR products by the use of primers that have one or two mismatches near the mutation, and thus allows rapid analysis of multiple samples for a known mutation. As demonstrated in Figure 2, parents could be identified appropriately as heterozygous carriers of the respective mutations. A recently born sibling of patient 1 was found to be free of the D215N mutation (not shown). 


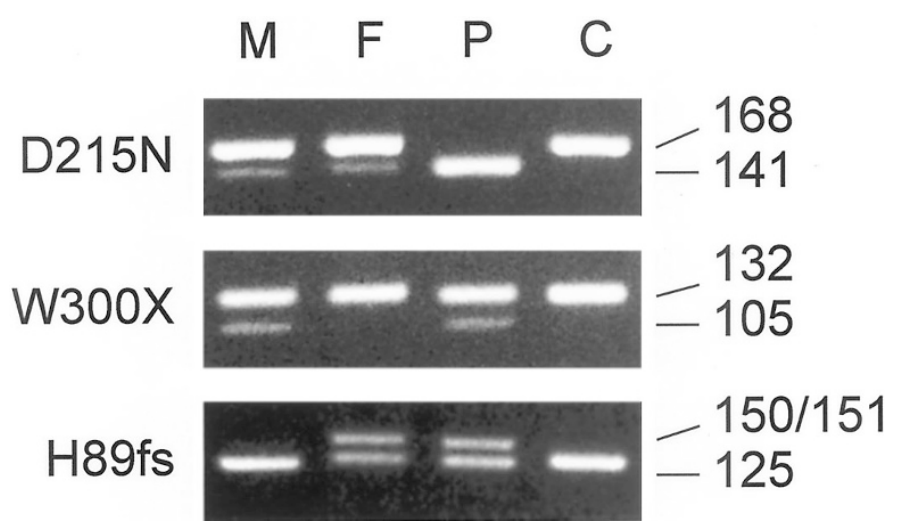

Figure 2. Detection of $P H K G 2$ mutations in family members by amplified created restriction sites. Ethidium bromide-stained electrophoretic DNA band patterns of mother $(M)$, father $(F)$, patient $(P)$, and a normal control $(C)$ are shown. Top, D215N mutation in family 1. Bcll cuts the 168-bp-long PCR product of the mutant allele into fragments of 141 and $27 \mathrm{bp}$, whereas the normal allele remains undigested. Middle, W300X mutation in family 2. BspHI cuts the 132-bp-long PCR product of the mutant allele into fragments of 105 and $27 \mathrm{bp}$, whereas the normal allele remains undigested. Bottom, H29fs(insC) mutation in family 2. XcmI cuts the 150 -bp-long PCR product of the normal allele into fragments of 125 and $25 \mathrm{bp}$, whereas the mutant allele (151 bp) remains undigested. The 25- and 27-bp fragment bands stain very faintly and are not shown.

\section{DISCUSSION}

The three novel mutations described here bring the total number of different PHKG2 mutations known to date to 11, found in 10 human patients and one animal mutant (Table 2). Of the 10 human patients, eight are homozygous for their mutations, being offspring of consanguineous parents, and two are compound heterozygous. The high proportion of females ( 9 of 10) and homozygotes in part reflects the fact that the first mutations in the autosomal PHKG2 gene were preferentially sought in these groups. However, three independent mutations have now been found among three patients from the small Norwegian population alone, suggesting that PHKG2 mutations may be more frequent than previously thought and should also be considered in male patients and in patients of nonconsanguineous descent.

All known PHKG2 mutations are point mutations, comprising missense (6 of 11), nonsense, splice-site, and frameshift mutations (single-nucleotide insertion or deletion). All but one affect the catalytic domain, which extends approximately between amino acids 24 and 290. The W300X mutation aborts the C-terminal 107 amino acids, which have regulatory function, harboring autoinhibitory sequences and binding sites for the subunits $\alpha$ and $\delta(2,21,22)$. This truncation probably destabilizes the holoenzyme. PHKG2 mutations may give rise to a particularly severe phenotype because $\gamma$ is the catalytic subunit whereas $\alpha$ and $\beta$ are regulatory.

In a longitudinal study of 41 Dutch and German patients with liver Phk deficiency (14), all of them male and most with established X-linked inheritance and therefore probably mutated in PHKA2, most displayed hepatomegaly (92\% of all), growth retardation (68\%) and delayed motor development $(52 \%)$, and elevated plasma cholesterol (76\%), triglycerides $(70 \%)$, and aminotransferases $(56 \%)$, whereas symptomatic hypoglycemia (12\%) and metabolic acidosis (2\%) were uncommon and splenomegaly was never present. Glucagon response was normal in 16 of 17 patients tested (14). Although fasting or exertional hypoglycemia, metabolic acidosis, or an abnormal glucagon response have been observed also in patients with documented PHKA2 or PHKB mutations $(3,7)$, these are clearly a minority in their genotype groups. In contrast, most PHKG2-mutant patients have hypoglycemic symptoms, and a high proportion display an impaired glucagon response or abnormal liver histology when analyzed (Table 2). Most strikingly, all three cases of liver Phk deficiency with childhood cirrhosis analyzed to date were found to carry PHKG2 mutations $(12,13)$.

All three patients described here had pronounced tendencies toward fasting hypoglycemia, pathologic glucagon responses, slight splenomegaly, and variable degrees of liver fibrosis, and one additionally displayed signs of incipient cirrhosis and portal hypertension. Together with the circumstance that all three are female and two have consanguineous parents, suggesting the involvement of an autosomal gene, these findings prompted an analysis of the PHKG2 gene where mutations indeed were found. Pronounced clinical and biochemical abnormalities in a case of Phk deficiency, with or without cirrhosis, therefore are a strong predictor for $P H K G 2$ mutations.

The phenotype of liver Phk deficiency caused by $P H K G 2$ mutations, more than that of PHKA2- or PHKB-mutant Phk

Table 2. PHKG2 mutations and associated phenotypes identified to date

\begin{tabular}{|c|c|c|}
\hline Genotype & Reference & Clinical features unusual in Phk deficiency \\
\hline $\mathrm{R} 44 \mathrm{X}$ & 12 (pt. B) & cirrhosis, hypoglycemia, lactic acidosis, weak glucagon response \\
\hline H89fs(insC) & 10 (pt. 1) & hypoglycemia, weak glucagon response \\
\hline L93fs(delC) & 12 (pt. A) & $\begin{array}{l}\text { cirrhosis, esophageal varices, ascites, adenomatous hepatocellular } \\
\text { hyperplasia, no glucagon response }\end{array}$ \\
\hline V106E & 10 (pt. 3) & fibrosis \\
\hline IVS4 $+1 \mathrm{G}>\mathrm{A}$ & 11 & fibrosis, splenomegaly, cholestasis \\
\hline H144Y/L229R & 13 & cirrhosis, hypoglycemia, lactic acidosis \\
\hline D215N & this study (pt. 1) & hypoglycemia, no glucagon response, fibrosis \\
\hline W300X/H89fs(insC) & this study (pt. 2) & hypoglycemia, no glucagon response, fibrosis \\
\hline
\end{tabular}

All patients had hepatomegaly and elevated aminotransferases. Growth retardation is reported for 8 of 10 , and muscle weakness for 3 of 10 . Hypoglycemia indicates hypoglycemic symptoms, documented low plasma glucose, or both. Where only one mutation is indicated as the genotype, patients are homozygous for the mutation. The D215N mutation is also found in the $g s d$ rat (10). The complete PHKG2 sequence is 406 amino acids long. 
deficiency, overlaps with and combines certain features of GSD I and III. The fasting hypoglycemia and impaired glucagon response are reminiscent of GSD I. However, GSD I is additionally characterized by hyperuricemia and hyperlactatemia whereas aminotransferases are usually normal and there is no fibrosis. Elevation of plasma aminotransferases, hepatic fibrosis, and potential for hepatic cirrhosis, on the other hand, resemble GSD III. Hepatomegaly, growth retardation, and hypoglycemia are features shared by all three disorders (1).

Our knowledge of the long-term outcome of liver Phk deficiency of all genotypes is still poor. The large majority of PHKA2- and PHKB-mutant patients improve substantially during the second decade of life, many becoming virtually asymptomatic although the enzymatic deficiency persists. However, there is little information on how they develop in later life. In five older Japanese patients of unspecified genotype, severe fibrosis and even cirrhosis were found (23), but it remains to be clarified whether this observation can be generalized and is applicable to other ethnic groups. To date, only two PHKG2mutant patients have been followed beyond the first decade of life. Both displayed the catch-up growth during puberty that is typical for other liver Phk-deficient patients. Otherwise, their courses differ substantially. Whereas the clinical and biochemical abnormalities have largely disappeared in one Norwegian patient at age 18 y (10), the other (Japanese) has developed severe cirrhosis, adenomatous hepatocellular hyperplasia, esophageal varices, and ascites at age 26 y (12). Although the present work has confirmed that PHKG2 mutations are associated with a higher risk of severe liver disease than PHKA2 or PHKB mutations, a larger number of patients of all genotypes need to be followed for a much longer time to establish the prognostic ranges and possibly the additional genetic and nongenetic factors that influence the outcome.

\section{CONCLUSIONS}

Patients with $P H K G 2$ mutations require a more active management than other Phk-deficient patients. They must be monitored for liver failure including portal hypertension. Their nutritional status must be closely followed. Frequent feeding, uncooked cornstarch supplementation, nighttime gastric tube feeding, or a combination of these will often be necessary to maintain normoglycemia. It is hoped that this will also improve the long-term prognosis regarding growth, as is the case in GSD I $(1,15,16)$, and liver histology.

Acknowledgments. The authors thank the families for their cooperation, and Drs. H. Rootwelt (Oslo), V. Worthington (London), D. Bali and Y.T. Chen (Durham, NC, U.S.A.) for amplified created restriction sites and enzyme determinations, respectively.

\section{REFERENCES}

1. Chen YT 2001 Glycogen storage diseases. In: Scriver CR, Beaudet AL, Sly WS, Valle D (eds) The Metabolic and Molecular Bases of Inherited Disease, 8th ed. McGraw-Hill, New York, pp 1521-1551

2. Kilimann MW 1997 Glycogen storage disease due to phosphorylase kinase deficiency. In: Swallow DM, Edwards YH (eds) Protein Dysfunction and Human Genetic Disease. BIOS Scientific Publishers, Oxford, pp 57-75

3. Burwinkel B, Amat L, Gray RG, Matsuo N, Muroya K, Narisawa K, Sokol RJ, Vilaseca MA, Kilimann MW 1998 Variability of biochemical and clinical phenotype in X-linked liver glycogenosis with mutations in the phosphorylase kinase PHKA2 gene. Hum Genet 102:423-429

4. Hirono H, Shoji Y, Takahashi T, Sato W, Takeda E, Nishijo T, Kuroda Y, Nishigaki T, Inui K, Takada G 1998 Mutational analyses in four Japanese families with X-linked liver phosphorylase kinase deficiency type 1 . J Inherit Metab Dis 21:846-852

5. Hendrickx J, Lee P, Keating JP, Carton D, Sadharwalla IB, Tuchman M, Baussan C, Willems PJ 1999 Complete genomic structure and mutational spectrum of PHKA2 in patients with X-linked liver glycogenosis type I and II. Am J Hum Genet 64:15411549

6. Rudolfova J, Slovackova R, Trbusek M, Peskova K, Stastna A, Kozak L 2001 Identification of three novel mutations in the PHKA2 gene in Czech patients with $\mathrm{X}$-linked liver glycogenosis. J Inherit Metab Dis 24:85-87

7. Burwinkel B, Maichele AJ, Aagenaes Ö, Bakker HD, Lerner A, Shin YS, Strachan JA, Kilimann MW 1997 Autosomal glycogenosis of liver and muscle due to phosphorylase kinase deficiency is caused by mutations in the phosphorylase kinase $\beta$ subunit $(P H K B)$. Hum Mol Genet 6:1109-1115

8. van den Berg IE, van Beurden EA, de Klerk JB, van Diggelen OP, Malingre HE, Boer MM, Berger R 1997 Autosomal recessive phosphorylase kinase deficiency in liver, caused by mutations in the gene encoding the $\beta$ subunit $(P H K B)$. Am J Hum Genet 61:539-546

9. Burwinkel B, Moses SW, Kilimann MW 1997 Phosphorylase-kinase-deficient liver glycogenosis with an unusual biochemical phenotype in blood cells associated with a missense mutation in the $\beta$ subunit gene (PHKB). Hum Genet 101:170-174

10. Maichele AJ, Burwinkel B, Maire I, Sövik O, Kilimann MW 1996 Mutations in the testis/liver isoform of the phosphorylase kinase $\gamma$ subunit (PHKG2) cause autosomal liver glycogenosis in the gsd rat and in humans. Nat Genet 14:337-340

11. van Beurden EA, de Graaf M, Wendel U, Gitzelmann R, Berger R, van den Berg IE 1997 Autosomal recessive liver phosphorylase kinase deficiency caused by a novel splice-site mutation in the gene encoding the liver gamma subunit (PHKG2). Biochem Biophys Res Commun 236:544-548

12. Burwinkel B, Shiomi S, Al-Zaben A, Kilimann MW 1998 Liver glycogenosis due to phosphorylase kinase deficiency: $P H K G 2$ gene structure and mutations associated with cirrhosis. Hum Mol Genet 7:149-154

13. Burwinkel B, Tanner MS, Kilimann MW 2000 Phosphorylase kinase deficient liver glycogenosis: progression to cirrhosis in infancy associated with PHKG2 mutations (H144Y and L225R). J Med Genet 37:376-377

14. Willems PJ, Gerver WJ, Berger R, Fernandes J 1990 The natural history of liver glycogenosis due to phosphorylase kinase deficiency: a longitudinal study of 41 patients. Eur J Pediatr 149:268-271

15. Wolfsdorf JI 2002 Bones benefit from better biochemical control in type 1 glycogen storage disease. J Pediatr 141:308-310

16. Schwahn B, Rauch F, Wendel U, Schönau E 2002 Low bone mass in glycogen storage disease type 1 is associated with reduced muscle force and poor metabolic control. J Pediatr 141:350-356

17. Sövik O, de Barsy T, Maehle B 1982 Phosphorylase kinase deficiency: severe glycogen storage disease with evidence of autosomal recessive mode of inheritance. Eur J Pediatr 139:210

18. Fernandes J, Huijing F, Van de Kamer JH 1969 A screening method for liver glycogen diseases. Arch Dis Child 44:311-317

19. Krebs EG 1966 Phosphorylase b kinase from rabbit muscle. Methods Enzymol 8:543-546

20. Tuchman M, Brown BI, Burke BA, Ulstrom RA 1986 Clinical and laboratory observations in a child with hepatic phosphorylase kinase deficiency. Metabolism 35:627-633

21. Calalb MB, Fox DT, Hanks SK 1992 Molecular cloning and enzymatic analysis of the rat homolog of "PhK- $\gamma \mathrm{T}$ ", an isoform of phosphorylase kinase catalytic subunit. J Biol Chem 267:1455-1463

22. Rice NA, Nadeau OW, Yang Q, Carlson GM 2002 The calmodulin-binding domain of the catalytic $\gamma$ subunit of phosphorylase kinase interacts with its inhibitory $\alpha$ subunit. J Biol Chem 277:14681-14687

23. Aikawa J, Ohura T, Iinuma K, Narisawa K 1997 Management of phosphorylase kinase deficiency - outcome and control of phosphorylase kinase deficiency. J Inherit Metab Dis 20:84

24. Hanks S, Quinn AM 1991 Protein kinase catalytic domain sequence database: identification of conserved features of primary structure and classification of family members. Methods Enzymol 200:38-62 